\title{
O uso em excesso do clonazepam: atribuições do farmacêutico no uso consciente do
}

\section{medicamento}

\author{
The overuse of clonazepam: pharmacist's role in the conscientious use of the drug \\ El uso excesivo de clonazepam: el papel del farmacéutico en el uso consciente del medicamento
}

Recebido: 25/11/2021 | Revisado: 30/11/2021 | Aceito: 03/12/2021 | Publicado: 19/12/2021

\section{Resumo}

Tabline D'avila Oliveira Pires ORCID: https://orcid.org/0000-0002-9079-4734 Faculdade de Palmas, Brasil E-mail: pirestabliny@gmail.com

Maykon Jhuly Martins de Paiva ORCID: https://orcid.org/0000-0002-6228-4550 Faculdade de Palmas, Brasil

Nos últimos anos, a busca e o consumo de drogas psicoativas tem aumentado gradativamente. Dentro desse rol, o medicamento clonazepam é o mais procurado nas farmácias brasileiras. Apesar desses medicamentos serem citados na literatura como um dos mais seguros, eles escondem graves problemas no seu manejo, pois as dificuldades inerentes a um quadro de dependência não prevalecem sobre os benefícios e, muitas vezes, passam despercebidas, devido à complexidade das situações envolvidas no tratamento dos pacientes. $\mathrm{O}$ trabalho teve como objetivo evidenciar o uso abusivo do clonazepam em conjuntura ao papel do profissional farmacêutico nesse cenário. A metodologia se tratou de uma revisão de literatura sistemática com levantamento de dados com 19 referências bibliográficas no período de 2010 a 2020 nas Bases de pesquisas científicas na área da saúde. Por meio do trabalho evidenciou-se as características farmacológicas, indicações, usos e contras do clonazepam, assim como a facilidade do seu uso abusivo e possíveis efeitos maléficos de sua dependência. O papel do farmacêutico é essencial devido seu conhecimento e atribuições no sentido de alertar os usuários sobre os riscos do potenciais efeitos colaterais e altas taxas de dependência.

Palavras-chave: Clonazepam; Uso abusivo; Farmacêutico.

\begin{abstract}
In recent years, the search for and consumption of psychoactive drugs has increased gradually. Within this list, the drug clonazepam is the most sought after in Brazilian pharmacies. Despite these drugs being cited in the literature as one of the safest, they hide serious problems in their management, because the difficulties inherent to a dependency situation do not prevail over the benefits and often go unnoticed due to the complexity of the situations involved in the treatment of patients. The objective of this study was to highlight the abusive use of clonazepam in conjunction with the role of the pharmaceutical professional in this scenario. The methodology was a systematic literature review with data survey with 19 bibliographic references in the period from 2010 to 2020 in the Bases of scientific research in the health area. Through this work, the pharmacological characteristics, indications, uses and cons of clonazepam were evidenced, as well as the ease of its abusive use and possible harmful effects of its dependence. The role of the pharmacist is essential due to his knowledge and attributions in order to warn users about the risks of potential side effects and high dependency rates.
\end{abstract}

Keywords: Clonazepam; Abuse; Pharmacist.

\section{Resumen}

En los últimos años, la búsqueda y el consumo de drogas psicoactivas ha aumentado gradualmente. Dentro de esta lista, el medicamento clonazepam es el más buscado en las farmacias brasileñas. Aunque estos fármacos se citan en la literatura como uno de los más seguros, esconden graves problemas en su manejo, ya que las dificultades inherentes a una situación de dependencia no prevalecen sobre los beneficios y a menudo pasan desapercibidos debido a la complejidad de las situaciones que implica el tratamiento de los pacientes. El objetivo de este estudio era poner de manifiesto el uso abusivo del clonazepam junto con el papel del profesional farmacéutico en este escenario. La metodología consiste en una revisión de la literatura sistemática con recogida de datos con 19 referencias bibliográficas en el período de 2010 a 2020 en las Bases de datos de investigación científica en el área de la salud. A través del trabajo se evidenciaron las características farmacológicas, indicaciones, usos y contras del clonazepam, así como la facilidad de su uso abusivo y los posibles efectos nocivos de su dependencia. El papel del farmacéutico es esencial debido a sus 
conocimientos y atribuciones para advertir a los usuarios sobre los riesgos de los posibles efectos secundarios y las altas tasas de dependencia.

Palabras clave: Clonazepam; Abuso; Farmacéutico.

\section{Introdução}

O consumo e a procura por drogas psicoativas têm aumentado gradativamente. Dentro desse rol, o medicamento clonazepam é o mais procurado nas farmácias brasileiras. Segundo dados da Agência Nacional de Vigilância Sanitária (ANVISA), o consumo no Brasil do princípio ativo do Rivotril@, medicamento de referência, aumentaram $22 \%$ em março e abril de 2020 em comparação ao mesmo período do ano de 2019, passando de 4,6 milhões para 5,6 milhões de caixas. O notável crescimento em pouco tempo leva a suspeitas de uso excessivo e desnecessário por especialistas (Sindusfarma, 2020).

O clonazepam pertence à classe farmacológica dos benzodiazepínicos, classe essa formada de medicamentos recomendados para terapêutica de patologias como: ansiedade, insônia, distúrbios de humor e outros quadros coligados a transtornos mentais e ao sistema nervoso central. Inúmeros estudos discorrem sobre o consumo de forma irracional ou exagerada de fármacos dessa classe dentro de um intervalo de tempo maior que seis meses pode ocasionar nos usuários condições clínicas de dependência química, física e/ou psicológica (Cruz, 2016).

Apesar desses medicamentos serem citados na literatura como um medicamento de uso seguro, eles escondem graves problemas no seu manejo, pois as dificuldades inerentes a um quadro de dependência não prevalecem sobre os benefícios e, muitas vezes, passam despercebidas, devido à complexidade das situações envolvidas no tratamento dos pacientes (Mendes, 2013).

A International Narcotics Control Board (INCB) informou em um relatório publicado em 2011 sobre o abuso sistemático de preparações farmacêuticas contendo clonazepam, bem como a incidência de excesso de prescrição desse medicamento pela facilidade de compra de prescrições em muitos países. No Brasil, os dados relativos à comercialização do clonazepam apontam para um consumo superior a doze milhões de unidades desse medicamento, entre os anos de 2008 e 2009 (Anvisa, 2011).

Os prescritores e os usuários encontram barreiras em propor protocolos que levem a redução do consumo do medicamento até atingir sua interrupção total, considerando que muitos usuários omitem ou negam sentir os efeitos indesejáveis do medicamento. Dessa forma, o papel do farmacêutico é marcante como um aliado do usuário durante sua reabilitação e a conscientização do uso racional do fármaco. Dentre suas múltiplas competências, o profissional farmacêutico se delega de avaliar, prevenir, reduzir ou minimizar o conflito da interação medicamentosa terapêutica, prevenindo a manifestação de novos problemas à saúde do usuário, além de minimizar custos para o sistema de saúde. Assim, satisfazendo uma necessidade social através do atendimento das necessidades individuais dos usuários (Brasil, 2014). Assim, se tem como objetivo evidenciar o uso abusivo do clonazepam e as contribuições do papel do profissional farmacêutico para o uso consciente do medicamento.

\section{Metodologia}

O presente trabalho é uma revisão sistemática e qualitativa acerca do uso de benzodiazepínicos, com particular ênfase nas propriedades do clonazepam, o seu uso excessivo e as contribuições do farmacêutico para o uso racional. Foi realizado através de coleta de dados com 20 referências bibliográficas entre 2005 e 2020, utilizando como descritores: clonazepam, abuso e dependência, e regulamentação farmacêutica na SCIELO (Scientific Online Library) e PUBMED (Public Medline), CAPES e Google Acadêmico. Para auxiliar na seleção do material bibliográfico foi aplicada a ferramenta PRISMA 2009 flow diagram. Figura 1. 
As revisões coletadas entre os meses de agosto a novembro de 2021, em português. Como critério de seleção, foram revisados artigos contendo dados bibliográficos abrangendo características, uso e abuso, cuidados farmacêuticos e outras informações específicas relevantes para o tema, um breve resumo dos dados referenciais está descrito no Quadro 1.

Figura 1 - Percurso de coleta de dados bibliográficos

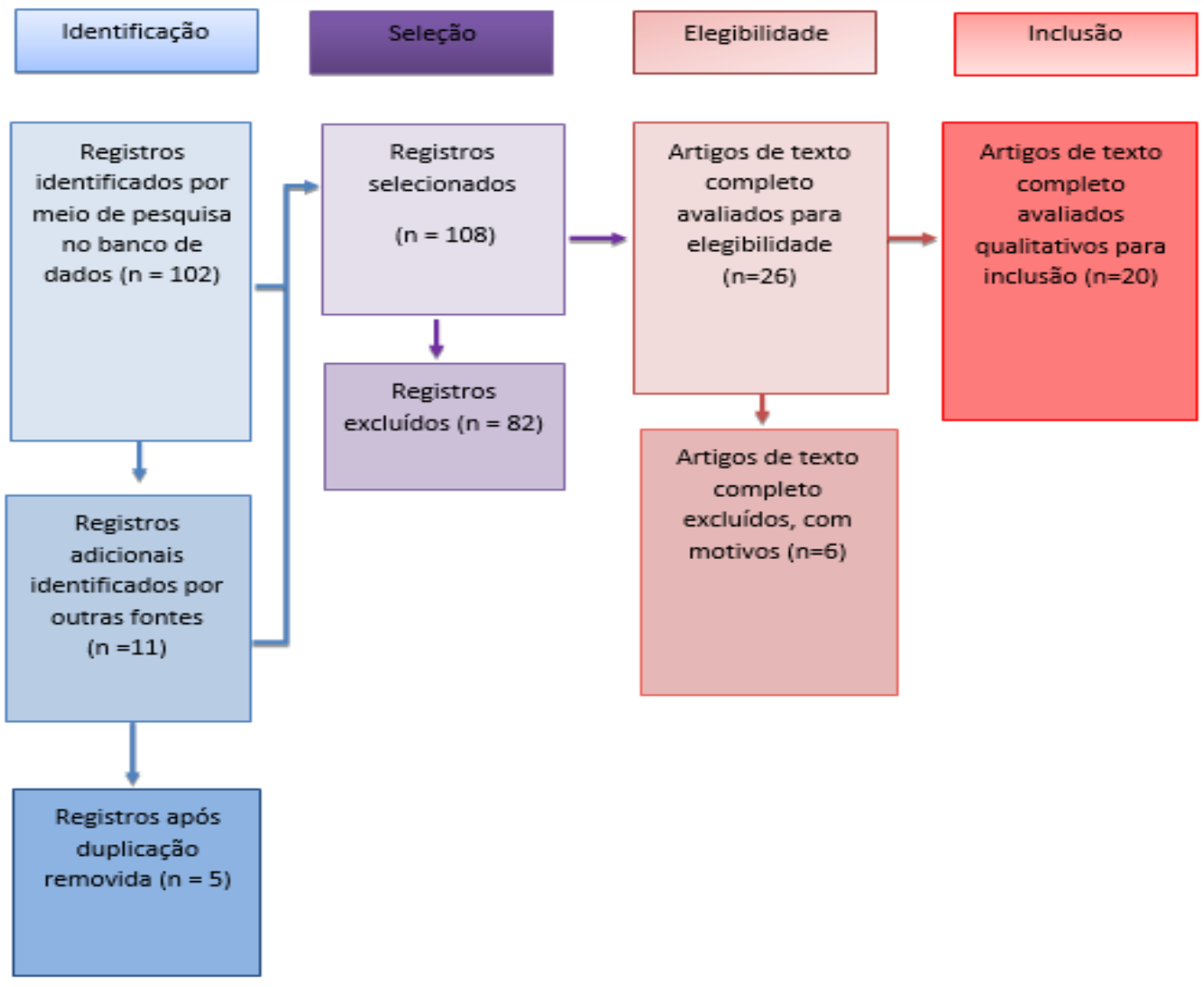

Fonte: Autores (2021).

\section{Resultados e Discussão}

A classe medicamentosa dos benzodiazepínicos teve sua origem na década de 50, proporcionado diversidade de compostos e consequentemente de usos. Tal classe é bastante conhecida pelos seus efeitos ansiolíticos, hipnóticos, sedativos e também como anticonvulsivantes. Devido sua farmacodinâmica, a classe apresenta efeitos anestésicos, relaxante muscular, vertentes da ansiedade como transtorno obsessivo compulsivo, usuários abstinentes ao álcool e também no estresse póstraumático (Mendonça \& Carvalho, 2005).

O clonazepam consiste em um benzodiazepínico derivado do nitrazepam, que através do processo de halogenação é possível obter-se sua estrutura química. Ele é considerado um "benzodiazepínico clássico", pois além de ser um dos que possuem estrutura molecular mais simples, também foi um dos primeiros a ser sintetizados em laboratório, juntamente com o diazepam, lorazepam, oxazepam, nitrazepam, flurazepam, bromazepam e clorazepato, medicamentos de mesma classe (Figura 2). 
Figura 2 - Estrutura molecular clonazepam

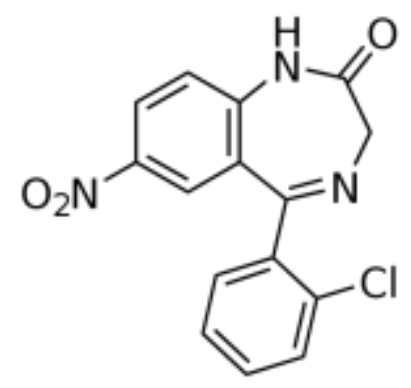

Fonte: Ferraz (2010).

\section{Farmacodinâmica e farmacocinética do clonazepam}

\section{Indicações terapêuticas e formas farmacêuticas}

Em especial, o clonazepam possui indicação para fobia social, transtornos do pânico, mania aguda, acatisia induzida por neuroléptico, ansiedade generalizada, redução transitória dos efeitos de discinesia tardia, insônia, crises epiléticas tônicoclônicas, ausências típicas e atípicas, crises mioclônicas e neuralgia do trigêmeo. O clonazepam também se manifesta de forma útil no tratamento das crises de ausência e convulsões mioclônicas em crianças (Barros \& Barros, 2010).

Abaixo segue as formas farmacêuticas comercializadas do clonazepam no Brasil. Quadro 1.

Quadro 1 - Formas Farmacêuticas do disponíveis do clonazepam

Comprimidos de $0,5 \mathrm{mg}$
Princípio ativo: clonazepam ................................................. $0,5 \mathrm{mg}$.
Excipientes: Lactose, estearato de magnésio, celulose microcristalina, amido de milho, povidona, corante amarelo e
talco.
Comprimidos de $2,0 \mathrm{mg}$
Princípio ativo: clonazepam ............................................ $2,0 \mathrm{mg}$.
Excipientes: Lactose, celulose microcristalina, amido de milho, povidona, talco e estearato de magnésio.
Solução oral de $2,5 \mathrm{mg} / \mathrm{mL}(1 \mathrm{gota}=0,1 \mathrm{mg}):$

Fonte: Ferraz (2010).

O clonazepam compartilha propriedades farmacodinâmicas com os benzodiazepínicos, incluindo as indicações clássicas ansiolíticas, sedativas, anticonvulsivantes, e relaxantes. Tal como acontece com os fármacos dessa classe sabe-se que esses efeitos sejam principalmente devidos à inibição pós-sináptica mediada pelo ácido gama-aminobutírico (GABA), embora vários estudos publicados também tenham sugerido um efeito do clonazepam em outro neurotrasmissor, a serotonina (Rang, Ritter \& Flower, 2016).

A absorção do clonazepam pela via oral chega a ser quase $100 \%$. As concentrações plasmáticas máximas são atingidas dentro de um intervalo de $1 \mathrm{~h}$ a 4 horas. A biodisponibilidade plena é de $90 \%$ dos tecidos. Os comprimidos de são bioequivalentes à solução oral em termos do grau (extensão) de absorção do fármaco, enquanto a taxa de absorção é ligeiramente mais lenta para os comprimidos, devido sua forma farmacêutica. As concentrações no estado estacionário (igualdade entre a taxa de eliminação e a taxa de biodisponibilidade) do clonazepam para um regime de uma vez ao dia durante 30 dias foram três vezes mais elevadas do que as obtidas com uma dose oral única (Goodman, 2012). 
A distribuição do clonazepam se dá rapidamente em vários órgãos e tecidos do corpo, sendo as estruturas cerebrais absorvidas preferencialmente. $\mathrm{O}$ volume médio de distribuição do fármaco é estimado em aproximadamente $3 \mathrm{~L} / \mathrm{kg}$. A meia-vida distribuída é de quase meia a uma hora. A ligação do clonazepam às proteínas plasmáticas está entre 82 e $86 \%$ (Telles Filho, 2011).

O metabolismo do clonazepam ocorre principalmente no fígado por dois mecanismos: oxidação, influenciada pela idade e hepatopatias, e conjugação que não sofre a interferência desses fatores (Leonardi, Azevedo \& Oliveira, 2017). Na família $3^{\mathrm{a}}$ os citocromos P-450 desenvolve um papel importante no metabolismo, em particular na redução de nitrogênio para metabólitos farmacologicamente inativos.

A genética também desempenha um importante papel no metabolismo. Os que são metabolizados por oxidação produzem metabólitos ativos, e os metabolizados por conjugação não produzem metabólitos ativos. Esses fatores relacionados ao metabolismo determinam à meia-vida plasmática do medicamento, isto é, o tempo decorrido entre a obtenção da concentração plasmática máxima e a metade desta. A excreção se faz através da conjugação com o ácido glicurônico (Albertino \& Moreira, 2017 apud Leonardi et al. 2017).

Sobre a eliminação do clonazepam, sabe-se que o tempo de meia-vida de eliminação é de 30 a 40 horas. Considera-se que a depuração renal é de $55 \mathrm{~mL} / \mathrm{min}$. As maiores frações dos metabólitos do fármaco são eliminados na urina, cerca de 50 a $70 \%$, sobre essa taxa apenas $2 \%$ da forma inalterada do fármaco é detectável. A eliminação nas fezes se dá em menor escala, podendo ser na forma livre e na forma de metabólito conjugado. Algumas literaturas sugerem que a farmacocinética de clonazepam é dose independente e pesquisas e ensaios clínicos científicos realizados em participantes voluntários com dose múltipla, mostraram que as concentrações plasmáticas do clonazepam são totalmente proporcionais à dose (Katzung \& Trevor, 2010).

\section{Interações medicamentosas}

A utilização do clonazepam pode ser em combinação com um ou mais medicamentos antiepilépticos. A probabilidade de uma interação farmacocinética com esses outros medicamentos é baixa. No entanto, adicionar um medicamento a farmacoterapia do paciente requer avaliação cuidadosa da resposta clínica do usuário, devido ao potencial de eventos adversos, como sedação e letargia. Nestes casos, a dose e concentração do medicameto deve ser ajustada para atingir os efeitos almejados (Nunes \& Bastos, 2016).

\section{Interações farmacocinéticas fármaco e fármaco}

A administração de fenitoína, fenobarbital, carbamazepina, lamotrigina e valproato (em dosagens menores) podem aumentar a depuração do clonazepam, fazendo com que reduza as concentrações plasmáticas do fármaco em até $38 \%$ durante o tratamento. clonazepam isoladamente não altera as enzimas responsáveis do seu metabolismo. As enzimas envolvidas no metabolismo não foram totalmente mapeadas e esclarecidas, mas sabe-se que incluem o rol da CYP3A4. Os inibidores da CYP3A4 (por exemplo, fluconazol) podem comprometer o metabolismo e por consequência obter-se a concentrações e efeitos do clonazepam exagerados (Anvisa, 2016).

\section{Interações farmacodinâmicas fármaco e fármaco}

A administração concomitante do clonazepam com ácido valproico pode levar crises epilépticas do tipo pequeno mal. Se o clonazepam for utilizado em conjunto com qualquer agente depressor de ação central, inclusive álcool os efeitos colaterais como sedação e depressão cardiorrespiratória podem ser aumentados. Os usuários em tratamento do fármaco devem evitar a ingestão de álcool. Apesar disso, "a ingestão de remédio com outra substância se torna comum, por diversos fatores, mas o 
principal é querer potencializar o medicamento é o que acontece como clonazepam e álcool" (Jacauna \& Rodrigues, 2021). Nos casos de tratamento combinado de medicamentos de ação central, a dosagem de cada medicamento deve ser ajustada, para obter efeito pleno (Anvisa, 2016).

\section{Interações fármaco e alimento}

Ainda não foram totalmente estabelecidas as interações do clonazepam com alimentos. Acredita-se que o suco de toranja abranda a atividade do citocromo P-450 3A4, que está envolvido no metabolismo de medicamento, e pode cooperar para o aumento das concentrações plasmáticas do fármaco e que a cafeína tem efeito antagônico durante o sono laboral (Porto, 2010).

\section{Contraindicações e efeitos causados pelo uso prolongados do Clonazepam}

O consumo do clonazepan é contraindicada a usuários com hipersensibilidade conhecida da molécula ou a qualquer substância dos excipientes do medicamento. Pode-se afirmar a contraindicação a usuários que possuam insuficiência respiratória grave ou comprometimento hepático grave, isso porque o clonazepam podem levar à ocorrência de encefalopatia hepática. O clonazepam na forma farmacêutica de comprimido está contraindicado para o tratamento de transtornos do pânico em usuários com histórico médico de apneia do sono, sendo preferencial o uso na forma líquida devida cinética ser mais rápida nos casos de crises (Mendes, 2013).

$\mathrm{O}$ uso superior a um período maior que seis meses do clonazepam podem desencadear dependência e tolerância à sustância, caso o uso dele seja exagerado e com um tempo maior que o supracitado os agravantes poderão ser muito maiores, necessitando de dosagens cada vez mais altas para obter-se o efeito desejado. Usuários com histórico de dependência química tanto a substâncias ilícitas quanto ao álcool tem maiores taxas de dependência ao fármaco. Deste modo, esses usuários precisam ser assistidos e observados com um olhar maior de cuidado, uma vez que os riscos de aumentar dependência pelo clonazepam são mais altos. Outro ponto a ser considerado está na interrupção do tratamento, visto que é um erro parar o uso do medicamento de maneira repentina, é coerente e certo um desmame contínuo com cerca de um quarto da dose em uso a cada 01 ou 02 semanas, a interrupção do tratamento sem que haja a diminuição de dosagem do medicamento, pode causar grandes chances de desenvolvimento de síndrome de abstinência. Assim poderá desenvolver sintomas psicóticos, distúrbio de comportamento, tremores, sudorese, agitação, dores musculares, alucinações, cefaleia, dores musculares, confusão mental, ansiedade e irritabilidade (Costa, Teixeira \& Silva, 2020)

\section{Regulamentação e os entraves do abuso do uso do clonazepam}

Segundo Portaria regulamentadora n. ${ }^{\circ}$ 344, de 12 de maio de 1998: A Notificação de Receita é personalizada e intransferível, devendo conter somente uma "B1" (psicotrópicas), deste Regulamento Técnico e de suas atualizações, ou um medicamento que as contenham. A Notificação de Receita "B", de cor azul, impressa sobre as despesas do profissional ou da instituição, terá validade por um período de 30 (trinta) dias contados a partir de sua emissão e somente dentro da Unidade Federativa que concedeu a numeração. A Notificação de Receita "B" poderá conter no máximo 5 (cinco) ampolas e, para as demais formas farmacêuticas, a quantidade para o tratamento correspondente no máximo a 60 (sessenta) dias exemplo é solução oral que a apresentação farmacêutica é de $2 \mathrm{mg} / \mathrm{mL}$ em que cada gota equivale a $0,1 \mathrm{~mL}$ (Araújo, 2015).

Por integrar o grupo das substâncias sujeitas ao controle especial, dispensadas apenas mediante receita médica, são exigidas normas em termos de prescrição e dispensação, apesar das evidências de abuso pelo público. Vendas imprecisas e prescrições inconsistentes de clonazepam permite que os usuários abusem delas e tenham a oportunidade de desenvolver consequentemente com maior facilidade tolerância e dependência. $\mathrm{O}$ uso de forma indiscriminada de clonazepam é de extrema importância devido ao seu impacto econômico e social envolvendo diversos aspectos (Dias, 2014). 
São inúmeros os casos de abuso e dependência correlacionados ao uso tanto terapêutico, quanto irracional de benzodiazepínicos. Sua suspensão abrupta pode causar reações de abstinência. Os sintomas relacionados à abstinência como já citado, incluem: ansiedade, agitação, irritabilidade, insônia, cefaléia, tremores, tontura, anorexia, náuseas, vômitos, diarréia, fraqueza, fotofobia, despersonalização e depressão. Esses sintomas podem surgir até uma semana após a retirada do medicamento. Dependem da meia-vida, da conversão em metabólitos ativos e respectivas meias-vidas (Silva, 2012).

\section{Papel do farmacêutico no uso consciente do medicamento}

O farmacêutico é o profissional que está inserido no cuidado ao paciente, participando ativamente da terapia medicamentosa, da promoção e/ou recuperação da saúde, exercendo suas atividades com autonomia para a tomada de decisões baseadas nos princípios éticos da profissão (CRF, 2019).

Dentro das atribuições desempenhadas pela profissão as sistematizações das intervenções farmacêuticas e a troca de informações dentro de um sistema no qual participam vários atores tem grande potencial de contribuição, seja a nível individual ou coletivo, para o uso racional de medicamentos. O farmacêutico dentro de suas competências, torna-se imprescindível ter: conhecimentos, atitudes e habilidades que permitam ao mesmo integrar-se à equipe de saúde e interagir mais com o paciente e a comunidade, contribuindo para a melhoria da qualidade de vida, em especial, no que se refere à otimização da farmacoterapia e o uso racional de medicamentos. As sistematizações das intervenções farmacêuticas e a troca de informações dentro de um sistema no qual participam vários atores tem grande potencial de contribuição, seja a nível individual ou coletivo, para o uso racional de medicamentos (Silva, 2012)

A importância do farmacêutico enquanto cidadão e profissional da saúde, na conscientização, prevenção e diminuição do uso indiscriminado clonazepam, pode se resumir em tomada de decisões e ações descritas no Quadro 2.

Quadro 2 - Atribuições do farmacêutico para conscientização e uso racional do clonazepam.

Implantar um CIM (Centro De Informações Sobre Medicamentos) para ser um facilitador de dúvidas, conscientizar, propagar informações relevantes imparciais, científicas e seguras aos usuários.

Integrar a equipe multiprofissional de atenção à saúde como um perito em medicamentos, discutindo, dando alternativas terapêuticas, possíveis reconciliações medicamentosas, informações sobre apresentações e quaisquer informações técnicas do clonazepam.

Orientar o usuário quanto ao uso rigoroso e correto, abrangendo os efeitos terapêuticos, posologia, interações com outras medicações, horários, doses máximas e mínimas, riscos e toxicidade do medicamento

Acompanhar os resultados do tratamento, pesquisa sobre reações adversas, realizar as intervenções, caso necessário, instigar se ocorreram erros de medicação e acompanhar se a adesão ao tratamento está sendo efetiva.

Fonte: Silva (2012)

Daí vem a importância do papel do profissional Farmacêutico nesse cenário orientando os usuários quanto ao uso racional do clonazepam, realizando uma correta dispensação e um acompanhamento farmacoterapêutico do tratamento, prestando um cuidado farmacêutico necessário, visando melhorar a qualidade de vida do usuário (Silva, 2012). Essas práticas por parte dos profissionais de farmácia, podem contribuir no combate do uso crônico e indiscriminado do Clonazepam (Andrade et al., 2020).

\section{Considerações Finais}

O uso abusivo do clonazepam parece envolver, além do usuário, os médicos que prescrevem o medicamento e os farmacêuticos que dispensam o medicamento. A falta de informação e o desconhecimento das consequências nefastas do 
consumo dessa droga por esses três atores (médicos, farmacêuticos e usuários) somam-se a uma série de outras questões abordadas nesse contexto. Conclui-se, portanto, que para o aprimoramento da conscientização do uso, é necessário o apoio multidisciplinar aos pacientes, ressaltando-se a importância dos farmacêuticos responsáveis pela correta e legal dispensação das prescrições. O farmacêutico deve usar seu conhecimento e atribuições conferidas no sentido de alertar os usuários sobre os riscos dos potenciais efeitos colaterais e altas taxas de dependência, também lembrando das doses terapêuticas e das doses inadequadas, assim, promover o uso racional e, consequentemente, melhorar a qualidade de vida dos usuários do medicamento.

Faz-se necessárias mais estudos dentro desta área para observar o impacto do uso do clonazepam no mundo e no Brasil e mais pesquisas dentro da área farmacêutica evidenciando o grande papel que o profissional farmacêutico desempenha no cenário da assistência voltada ao uso racional de bcaenzodiazepínicos. Realizando pesquisa de campo que mostre a realidade do farmacêutico frente às orientações uso da medicação.

\section{Referências}

Albertino, S. \& Moreira Filho, P. F. (2000) Benzodiazepínicos: atualidades. Rev. bras. med. otorrinolaringol, 25-7. https://pesquisa.bvsalud.org/portal/resource/pt/lil-258168

Andrade, S. M. de, Cunha, M. A., Pereira Júnior, J. L., Maciel, A. L. de S., Santana, L. S. O. S., Carvalho, R. O., \& Oliveira, E. H. de. (2020). Uso crônico e indiscriminado de benzodiazepínicos: uma revisão de literatura. Research, Society and Development, 9(7), e317973954. https://doi.org/10.33448/rsd-v9i7.3954

Araújo, P. L. D. (2016). Associação do uso prolongado de benzodiazepínicos com o aumento do risco de demência em idosos: uma revisão bibliográfica. Monografia de Curso de Pós-graduação Lato Sensu. Universidade Do Extremo Sul Catarinense - UNESC. Criciúma - SC. http://repositorio.unesc.net/handle/1/4007

Barros, E., \& Barros, H. M. (2009). Medicamentos na prática clínica. Artmed Editora.

Costa, R. C. A, Teixeira, D. A \& da Silva, M. H. (2020), O uso indiscriminado de clonazepam e a importância da Assistência de enfermagem. Revista Saúde dos Vales. V.1 - N.1. Revistas institucional da UNIPAC- Teófilo Otoni - MG. https://revistas.unipacto.com.br/storage/publicacoes/2020/458_ o_uso_indiscriminado_de_clonazepam_e_a_importancia_da_assistencia_de_e.pdf

Conselho Regional De Farmácia do Estado de São Paulo (2019. Departamento de Apoio Técnico e Educação Permanente. Comissão Assessora de Farmácia Clínica. Farmácia Clínica. / Conselho Regional de Farmácia do Estado de São Paulo. - São Paulo: Conselho Regional de Farmácia do Estado de São Paulo, (2a ed.).

Cruz, N. L. D. M. (2016). Clonazepam, um campeão de vendas no Brasil. Por quê? Dissertação (Mestrado) - Universidade Federal de São Paulo (UNIFESP), São Paulo, SP. https://repositorio.unifesp.br/handle/11600/41881

Ferraz, J. A. M. B. (2010). Efeito do substituinte “- F” na interacção "Fármaco-Receptor” em Benzodiazepinas (Doctoral dissertation, [sn]). https://bdigital.ufp.pt/handle/10284/1654

Forsan, M. A. (2010). O uso indiscriminado de benzodiazepínicos: uma análise crítica das práticas de prescrição, dispensação e uso prolongado. Monografia - Universidade Federal de Minas Gerais, Campos Gerais. https://repositorio.ufmg.br/handle/1843/BUOS-9CLGH4

Goodman, L. (2010). Goodman \& Gilman: as bases farmacológicas da terapêutica (10a ed.), MGraw Hill.

Jacauna, J. S. P., \& Rodrigues Junior, O. M. (2021). Cuidados farmacológicos na interação medicamentosa: clonazepam com álcool. Research, Society and Development, 10(15), e226101522771. https://doi.org/10.33448/rsd-v10i15.22771

Katzung, B. G., \& Trevor, A. J. (2017). Farmacologia Básica e Clínica-13. McGraw Hill Brasil.

Leite, F.M. (2015). Diminuição do uso de benzodiazepínicos na penitenciária de Santa Vitória. Monografia. Universidade Federal de Minas Gerais, Santa Vitória - MG. https://www.nescon.medicina.ufmg.br/biblioteca/imagem/diminuicao-uso-benzodiazepinicos-penitenciaria-vitoria.pdf

Leonardi, J. G, Azevedo, B. M \& Oliveira, A. C. C (2017). Benzodiazepínicos e seus efeitos no sistema nervoso central. Revista Saúde em Foco, no 9. https://portal.unisepe.com.br/unifia/wp-content/uploads/sites/10001/2018/06/076_benzodiazepinicos.pdf

Ministério da Saúde; 2014. Cuidado Farmacêutico na Atenção Básica. Caderno 4: resultados do processo de implantação do Cuidado Farmacêutico no município de Curitiba. Brasília: Ministério da Saúde.

Mendes K.C.C (2013). O uso prolongado de benzodiazepínicos - uma revisão integrativa. Monografia - Universidade Federal de Minas Gerais, Pompéu. https://www.nescon.medicina.ufmg.br/biblioteca/imagem/4077.pdf

Nunes, B. S., \& Bastos, F. M. (2016). Efeitos colaterais atribuídos ao uso indevido e prolongado de benzodiazepínicos. Saúde \& ciência em ação, 2(2), 71-82. http://revistas.unifan.edu.br/index.php/RevistaICS/article/view/234/177

Porto C.C (2011). Interação medicamentosa. Guanabara Koogan. 
Research, Society and Development, v. 10, n. 16, e315101623880, 2021

(CC BY 4.0) | ISSN 2525-3409 | DOI: http://dx.doi.org/10.33448/rsd-v10i16.23880

Rang, R., Ritter, J. M., Flower, R. J., \& Henderson, G. (2015). Rang \& dale farmacologia. Elsevier Brasil.

Telles Filho, P. C. P., Chagas, A. R. D., Pinheiro, M. L. P., Lima, A. M. D. J., \& Durão, A. M. S. (2011). Utilização de benzodiazepínicos por idosos de uma estratégia de saúde da família: implicações para enfermagem. Escola Anna Nery, 15, 581-586. http://www.redalyc.org/articulo.oa?Id=127719485020 